Ann. Sci. forest., 1979, 36 (1), 1-12.

\title{
« Matorrals » méditerranéens et «Chaparrals » californiens Quelques aspects comparatifs de leur dynamique, de leurs structures et de leur signification écologique
}

\author{
P. QUEZEL (1) \\ Faculté des Sciences et Techniques de St-Jérôme* \\ * Botanique et Ecologie méditerranéenne, rue Henri-Poincarré, \\ 13397 Marseille Cedex 4
}

\section{Résumé}

Matorrals et Chaparrals offrent d'indiscutables homologies physionomiques et même floristiques. Des différences appréciables apparaissent cependant si l'on essaie d'interpréter la valeur dynamique et les structures de ces deux types de végétation. Il est ainsi possible de rendre compte, au moins en partie, des divergences très nettes qui apparaissent entre les auteurs de l'ancien monde et ceux de Californie à propos de leur valeur écologique et de leur modalité d'utilisation.

De nombreux auteurs ont souligné, déjà depuis longtemps, les affinités au moins physionomique et climatique existant à la surface du globe entre diverses zones caractérisées par un bioclimat de type méditerranéen. Le récent ouvrage de F. Di Castri et H. A. Mooney (1973) a d'ailleurs été consacré à cette question, et en introduction dans ce même volume A. W. Johnson analyse le concept de « convergence des écosystèmes $》$. II n'est pas douteux toutefois que c'est entre le monde circum-méditerranéen et la Californie que ces analogies ont été le plus souvent évoquées.

Certes, tous les auteurs sont loin d'être d'accord, et en particulier l'appréciation des caractères permettant de définir clairement le climat méditerranéen est assez différente entre les auteurs de l'ancien monde et ceux du nouveau monde. Actuellement, les critères retenus par Bagnouls et Gaussen (1953) puis par Emberger (1952, 1955) et précisés par Sauvage (1963) Nahal (1976) et Daget (1977) en particulier, sont en dépit de certaines différences, fondamentalement compatibles entre eux et ont tendance à être acceptés par tous les bioclimatologistes du pourtour méditerranéen. La prépondérance de la période de sécheresse estivale sur les autres facteurs climatiques (répartition des précipitations durant les autres saisons et températures minima en particulier) est, de ce fait déterminante, et a permis à Daget (1977) de définir son « aire isoclimatique méditerranéenne».

(1) Professeur à la Faculté des Sciences et Techniques de Marseille, St-Jérôme. 
Par contre, pour les Californiens, comme l'a indiqué H. Aschmann (1973) ( ${ }^{1}$ ) l'appréciation du climat méditerranéen est beaucoup plus restrictive, ce qui amène cet auteur en particulier à exclure du « vrai climat méditerranéen » de vastes zones européennes, où la végétation quant à elle appartient indiscutablement au « monde méditerranéen », le Languedoc, la majeure partie du littoral italien yougoslave et albanais en particulier.

Au niveau de la végétation, les analogies au moins physionomiques, souvent soulignées ont été précisées en particulier par P. J. Einke $(1965,1973)$ et établies au niveau de la flore par P. H. Raven (1971).

Nous ne voudrions pas revenir ici sur ces questions actuellement bien connues, mais nous souhaiterions toutefois à la suite d'un trop bref séjour en Californie méridionale et en Arizona tenter de dégager quelques idées générales sur la dynamique et les problèmes de sauvegarde ef d'amélioration que posent divers écosystèmes de type méditerranéen tant en Californie que dans l'ancien monde. L'accent sera d'ailleurs mis sur les écosystèmes de type maquis-garrigue ou chapparral (fruticée sclérophylle).

II est bien évident que l'on ne peut que souligner les singulières convergences physionomiques, mais aussi parfois floristiques existant entre les maquis et garrigues du bassin méditerranéen, pour lesquelles d'ailleurs le terme de « matorral » est de plus en plus souvent proposé (Sauvage, 1961 ; Tomaselli, 1976) et le « chaparral » californien. P. J. Zinke (loc. cit.) cite plusieurs vicariances physionomiques, d'ailleurs parfois discutables, mais il est certain que l'on ne peut être que frappé par le rôle des chênes sclérophylles, par celui des Rhamnacées (Rhamnus, Ceanothus) des Ericacées (Arbutus, Arctostaphylos) des Thérébinthacées (Rhus. Pistacia) dans ces formations aussi bien dans l'ancien monde que dans le nouveau. Les homologies apparaissant également, en particulier entre les genres Erica et Adenostoma, voire Eriogonum ou encore Cistus, Salvia, Encelia, sont aussi évidentes.

Ces ensembles floristiques ont sensiblement la même signification biogéographique dans les deux régions, puisqu'ils participent à la fois du fond holarctique et du fond tropical, mais il n'est pas douteux qu'en Californie, pour des raisons historiques évidentes (Axelrod, 1973), les taxons archaïques se sont mieux conservés, et le rôle du fond tropical est nettement plus important. N'oublions pas en effet qu'il existe toujours là un large contact entre flores méditerranéennes et tropicales, en particulier par l'intermédiaire des semi-déserts actuels à Mimosacées et à Cesalpiniacées, alors qu'il n'en est plus de même en Afrique et au Proche-Orient où ce contact $a$ pratiquement disparu, effacé depuis l'Holocène par le développement de vastes zones désertiques (Quézel, 1977).

La mise en parallèle des structures de végétation reste cependant difficile, car si dans l'ancien monde les techniques d'analyse phyto-écologique descriptives ont régulièrement progressé et bénéficié de synthèses phytosociologiques, mais aussi de la mise au point de nombreuses autres techniques : études quantitatives (cf. Gounot, 1969), méthode des groupes écologiques (Duvigneaud, 1946), en Californie I'on est resté à des interprétations strictement physionomiques des structures de végétation. Finalement peu de choses ont changé entre le travail de Cooper (1922) et celui

( ${ }^{1}$ ) Plus de $60 \%$ de pluies tombant en hiver et moyenne des minima du mois le plus froid supérieure pour la plupart des auteurs à $3^{\circ} \mathrm{C}$. 
tout récent de Barbour et Major (1977) quant à l'appréciation en particulier des divers types de chaparrals. De même, si divers auteurs étrangers ont tenté d'appliquer à la Californie leurs méthodes (Emberger, 1955 ; Gaussen, 1954) il faut bien avouer qu'ils n'ont guère été suivis par les scientifiques locaux, et l'on en est encore à attendre une analyse précise de la végétation aussi bien qu'une interprétation altitudinale compréhensive des étages de végétation.

\section{Les problèmes dynamiques}

Sur le pourtour de la Méditerranée, tous les phytogéographes considèrent ou ont considéré, à de très rares exceptions près, que les paysages du matorral s'inscrivent dans une série dynamique évolutive éventuelle, dont l'aboutissement climacique est à peu près constamment la forêt, qu'il s'agisse de forêt dense sclérophylle, caducifoliée, ou de forêt de conifères parfois clairsemés. Sans entrer ici dans les discussions relatives à la notion de climax, reconnaissons cependant qu'il est raisonnablement possible d'admettre, mais aussi de prouver, que dans un laps de temps suffisant et dans des conditions normales d'exploitation par l'homme, les matorrals sont susceptibles d'évoluer vers les formations forestières. II convient cependant de signaler que, depuis quelques années, divers auteurs (Ehrig, 1973 ; Quezel, 1976 ; Tomaselli, 1976) envisagent la possibilité d'existence de matorrals denses et élevés climaciques ou subclimaciques. C'est le cas particulièrement pour certains maquis à Erica arborea de Corse ou ceux à Arbutus andrachne de Turquie méditerranéenne, où l'installation de jeunes individus de Quercus ou de Pinus paraît bien difficile à envisager dans les conditions actuelles. II n'en reste pas moins que dans l'immense majorité des cas, et sur la quasi-totalité des surfaces, le passage du matorral à la forêt est possible ou au moins envisageable.

En Californie par contre, phytogéographes et écologistes considèrent que les chaparrals constituent très généralement des groupements climaciques, dont la transformation en forêts n'est pas envisageable (Cooper, 1922 ; Campbell et al., 1947 ; Sampson, 1944).

En Californie méridionale, les chaparrals occupent effectivement à eux seuls, une zone altitudinale importante et s'étendent approximativement entre le niveau de la mer et 800-1000 m sauf toutefois à proximité des cours d'eau et dans les thalwegs où se développent soit la forêt riveraine à Platanus racemosa, Populus trichocarpa, Alnus rhombifolia, Cercis occidentalis, voire à Pseudotsuga macrocarpa, dans ou à proximité immédiate du lit mineur à nappe phréatique proche, soit la forêt à Chênes sclérophylles où domine Quercus agrifolia, dans le lit majeur et les thalwegs ennoyés de sédiments (Rowe, 1963). Au-dessus de ces altitudes, ils existent encore, mais en tant que stades de dégradation des forêts de Conifères et en particulier des forêts de Pinus ponderosa souvent associé à $P$. lambertiana et $P$. jeffreyi.

Les chaparrals s'observent encore, au moins en exposition sud, jusqu'à plus de 2000-2 $300 \mathrm{~m}$ et offrent des rapports évidents suivant les régions et les expositions, avec les formations à Abies concolor, ou au sud, et dans les régions moins arrosées avec les forêts claires à Pinus monophylla et Juniperus californica.

Il est bien évident que en fonction de l'altitude, des substrats, et aussi des séries de végétation auxquelles ils s'intègrent éventuellement, les chaparrals offrent des structures floristiques fort différentes. 
En fait, il apparaît donc que si, en région méditerranéenne les matorrals représentent très généralement des stades de régression des écosystèmes forestiers, en Californie par contre cela n'est pas évident, en particulier au-dessous de $1000 \mathrm{~m}$, les chaparrals étant alors en dehors des thalwegs, considérés comme climaciques.

Il convient de s'interroger sur la signification exacte de ces dissemblances et de chercher éventuellement à en trouver les raisons.

Si l'on s'adresse bien sûr à des régicns climatiquement analogues comme c'est en particulier le cas pour la Californie méridionale et le Liban ou encore le Maroc atlantique, il n'est pas douteux que l'on est frappé, en Californie au-dessous de $800 \mathrm{~m}$, par la rareté des écosystèmes forestiers et par le développement considérable des paysages de chaparral, alors que l'impact humain n'est pas a priori important, et de toutes façons, infiniment moindre quant à la présence humaine et à l'intensité du pâturage, que dans l'ancien monde. Les facteurs édaphiques n'expliquent pas non plus ces différences et l'on est bien obligé de chercher ailleurs une solution.

Pour tous les auteurs américains, le chaparral constitue un écosystème spécial, étroitement lié à l'apparition périodique d'incendies, où le développement des buissons sempervirents s'effectue sur un cycle progressif durant une cinquantaine d'années, pour entrer ensuite nettement en régression du point de vue de la productivité (Hanes, 1971), et qui en même temps s'appauvrit du point de vue de son cortège floristique tout en conservant un recouvrement très élevé, la hauteur des végétaux stagnant elle aussi et n'excédant pas 2 à $4 \mathrm{~m}$ suivant les cas. Aucun élément franchement arborescent ne s'installe par ailleurs, et la seule possibilité d'évolution reste le passage généralement accidentel du feu qui détermine un nouveau cycle de végétation. Remarquons encore que très souvent, comme l'a montré L. Debano (1969), s'organise à la surface du sol, un horizon minéral non mouillable qui perturbe profondément l'équilibre hydrique de l'écosystème.

L'on reste malgré tout étonné, en arrivant d'Europe ou d'Afrique du Nord de voir se constituer un écosystème aussi singulier, dépourvu a priori d'avenir forestier, alors que dans l'ancien monde où les incendies de forêts et de maquis constituent un phénomène sans doute aussi fréquent qu'en Californie, ils n'entravent pas, sauf s'ils se succèdent de trop près, la réinstallation d'un couvert forestier.

Faut-il alors faire intervenir les structures végétales même de l'écosystème ?

Les Chênes sclérophylles sont, remarquons-le, infiniment moins nombreux et écologiquement moins diversifiés dans l'ancien monde où, en région méditerranéenne, ils sont représentés par quatre ou cinq espèces tout au plus, essentiellement localisées aux élages thermo et eu-méditerranéens, alors qu'il en existe une douzaine en Californie sans compter les genres Castanopsis et Lithocarpus, et qu'ils y prospèrent depuis le niveau de la mer jusqu'à la limite supérieure des forêts.

Les pins de basse altitude sont par contre plus nombreux dans la région méditerranéenne de l'ancien monde (cinq espèces) qu'en Californie méridionale (trois espèces). Ils sont surtout infiniment plus dynamiques puisque le Pin de Monterey (Pinus radiata), le Pin de Torrey (Pinus torreyana) et Pinus muricata n'y forment que des peuplements restreints. On s'interroge d'ailleurs sur les raisons de ce phénomène d'autant que Pinus radiata s'est révélé particulièrement dynamique dans d'autres régions à climat méditerranéen et en particulier au Chili.

Très singulièrement encore, les forestiers californiens n'envisagent guère d'utiliser leurs essences autochtones pour d'éventuels reboisements, alors que les essais sur 
Pinus halepensis, en forêt expérimentale de San Dimas par exemple, se sont révélés fort prometteurs.

Faut-il admettre que l'impossibilité apparente que présentent les écosystèmes de chaparral à évoluer vers des écosystèmes forestiers sont liés, en Californie, malgré la présence de nombreuses essences sclérophylles, au manque de dynamisme, voire à l'absence en de nombreuses localités de représentants des genres Pinus surtout, mais aussi Cupressus, qui forment dans l'ancien monde, des étapes pré-climaciques voire climaciques, en principe facilement réalisables sur le terrain, et souvent indispensables à l'installation de l'essence de feuillus climax!

Ełant donné la richesse du fond floristique californien cette idée peut évidemment choquer, mais elle n'est pas à repousser a priori et mériterait d'être approfondie.

L'on peut aussi penser que c'est la nature même des composantes végétales de l'écosystème chaparral qui est responsable de ce phénomène, en particulier pour des raisons chimiques et physico-chimiques (Mc Pherson et Muller, 1969).

Remarquons encore que la compréhension du terme «forêt» peut également constituer une difficulté supplémentaire, puisqu'actuellement dans l'ancien monde l'on parle de forêt pour des formations de plus de $2 \mathrm{~m}$, à condition que les troncs soient bien individualisés (Tomaselli, 1976) et que si les forestiers californiens adoptaient eux aussi cette définition, certains chaparrals, à Quercus dumosa en particulier, voire à Rhus ovata, mériteraient d'être rangés sous ce vocable.

\section{Structures et morphologie}

Une autre série de différences apparaît entre les matorrals méditerranéens et les chaparrals californiens au niveau des structures de végétation. En effet, sur le pourtour de la méditerranée, les formations sclérophylles offrent une diversité physionomique et au niveau des types biologiques qui les constituent, une héiérogénéité bien plus grande que leurs homologues californiennes. Il apparaît en effet qu'en Californie, les chaparrals sont essentiellement constitués par des chaméphytes érigés et des nanophanérophytes, du moins lorsqu'ils ont atteint leur stade de maturité. Les chaméphytes prostrés ou rampants sont peu nombreux, et à ce même stade de développement, les hémicryptophytes font à peu près défaut. Ces derniers existent bien dans les stades initiaux survenant après les incendies (Hanes, 1977), mais ils jouent semblet-il un rôle plus discret que les thérophytes qui disparaissent eux aussi progressivement et assez rapidement au cours du développement du chaparral. Ce phénomène est bien indiqué par Mooney et Parson (1973, pp. 96-98) qui insistent précisément sur l'importance de ces types annuels qualifiés de « annual fire-type » dont le dynamisme a été suivi par Horton et Kraebel (1955). Au niveau des chaparrals âgés, ces mêmes espèces se localisent uniquement au bord des chemins ou dans les zones perturbées.

En fait ce phénomène paraît répondre au niveau des structures de végétation à diverses particularités.

La prédominance des types érigés dans le chaparral est tout d'abord importante, puisqu'elle conditionne rapidement une couverture totale par la strate arbustive, mais aussi nous y reviendrons plus loin, parce qu'elle ne constitue pas a priori un facteur 
décisif contre les phénomènes d'érosion. L'absence totale_ou quasi-totale de chaméphytes nains prostrés et rampants, voire en coussinet, qui jouent un rôle si important dans les matorrals circum-méditerranéens est en effet un des caractères majeurs des chaparrals de Californie méridionale où ne se retrouvent pas en particulier les équivalents (à de rares exceptions près) des représentants des genres Dorycnium, Genista (p. p.), Sarcopoterium, Hypericum, Fumana, Helianthemum, Erica (p. p.), Lithospermum, Lavandula, Rosmarinus, Satureia, Teucrium, Thymus, Globularia, etc.

L'absence à peu près totale dans les chaparrals adultes ou sénescents, d'hémicryptophytes et bien sûr essentiellement de Graminées ou de Cypéracées, est une autre différence importante entre matorrals et chaparrals. En effet, bien que plus largement présent sur le pourtour méditerranéen, après les incendies et dans les stades initiaux de formation des fruticées sclérophylles, ce type biologique persiste à peu près toujours en quantité appréciable voire souvent importante dans les matorrals circum-méditerranéens âgés. C'est en particulier le cas pour les représentants des genres Festuca, Dactylis, Melica, Brachypodium, Agrostis, Stipa, Agropyron, Ampelodesmos, etc, et ceci aussi bien en France méditerranéenne qu'en Afrique du Nord par exemple, mais bien entendu avec des espèces parfois différentes. De même dans les portions les plus chaudes du bassin méditerranéen, étage thermo-méditerranéen en particulier (Quezel, 1976), les Andropogonées vivaces jouent un rôle de premier plan dans la dynamique de la végétation, alors que ces dernières sont pratiquement absentes des chaparrals californiens.

Les espèces annuelles qui, rappelons le, sont mieux représentées en région circum-méditerranéenne qu'en Californie, comme le montre le tableau ci-dessous,

$\%$ d'espèces annuelles dans la flore :

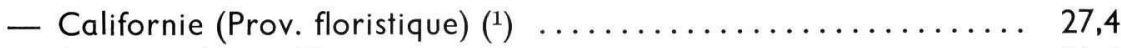

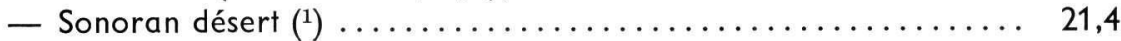

— Région méditerranéenne d'Afrique du Nord $\left({ }^{2}\right) \ldots \ldots \ldots \ldots \ldots .49$

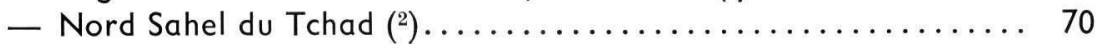

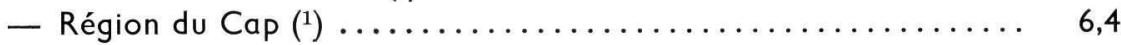

posent également d'intéressants problèmes. Ce type biologique, sur le pourtour méditerranéen comme en Californie, se développe après les incendies et constitue des pelouses à annuelles constituant le stade initial du schéma dynamique conduisant à la reconstitution des fruticées sclérophylles. Elles individualisent dans l'ancien monde, à ce moment, des types de végétation bien spéciaux, différents en fonction du substrat et des conditions bioclimatiques, dont les structures phytosociologiques sont bien connues. Il en est sans doute de même en Californie mais aucune étude de ce type n'y a été réalisée.

La reconstitution de la couverture arbustive détermine partout leur disparition au moins partielle, mais elles continuent néanmoins à jouer un certain rôle dans les maquis et garrigues circum-méditerranéennes, comme le montre clairement l'analyse phytosociologique de ces formations, alors qu'elles paraissent disparaître totalement dans les chaparrals, mais ici encore des informations précises font défaut.

(1) Goldblatt (1977)

(2) Quezel (1977). 


\section{Valeur écologique}

Des divergences considérables apparaissent encore dans l'appréciation de la valeur écologique des fruticées sclérophylles dans l'ancien monde et en Californie méridionale.

En effet, tous les écologistes des pays circum-méditerranéens sont conscients du rôle des maquis et garrigues dans les phénomènes de lutte contre l'érosion et de régulation du régime des eaux. C'est d'ailleurs pour ces raisons, qu'à la suite du rapport de Tomaselli (1976) une motion demandant la sauvegarde et le maintien des maquis et garrigues méditerranéens a été prise par les pays du Conseil de l'Europe.

En Californie et en Arizona par contre, à la suite d'études souvent très sophistiquées, les conclusions des écologistes et des aménageurs sont beaucoup plus nuancées. En effet comme cela a été montré à la forêt expérimentale de San Dimas (Mooney et Parson, 1973), mais aussi en divers autres points de Californie méridionale et encore en Arizona dans la forêt de Tonto, la conversion des matorrals en pelouses réduit d'une façon évidente les phénomènes d'érosion et améliore le bilan hydrique du sol, du moins sur les pentes dont l'inclinaison est inférieure à environ 40 p. 100 (Debano et Rice, 1971 ; E. Conrad et L. Debano, 1974), mais accroît par contre nettement audessus de ce taux de pente les chances de glissement de terrain (E. Corbett et R. Rice, 1966 ; Rice, 1973). Son effet est également bénéfique vis-à-vis des incendies (T. Brown et R. Boster, 1974).

On peut a priori s'étonner de voir proposer dans des régions aussi semblables par leur climat, et à première vue homologues par leur végétation, des interprétations aussi différentes quant à la valeur écologique des fruticées sclérophylles. On doit bien entendu s'interroger sur le bien fondé de ces conclusions, mais la rigueur de l'expérimentation poursuivie en Californie méridionale, pas plus que les observations convergentes réalisées dans le bassin méditerranéen depuis de très longues années, ne peuvent être mises en doute. Il convient dès lors de chercher les raisons de ces divergences.

Remarquons tout d'abord qu'en région méditerranéenne, un très long passé de pratiques pastorales et d'intenses influences humaines ont réalisé « de facto »la conversion en pâtures, de nombreuses surfaces boisées ou de maquis et garrigues, et il s'est établi très tôt un équilibre plus ou moins stable entre la forêt, les pâturages et les cultures (silva-saltus-ager Kunholtz-Lordat, 1938), équilibre qui a subsisté jusqu'au début de ce siècle et qui résultait d'un choix empirique mais généralement fort sage des populations locales, à propos de l'utilisation des sols. En Californie, les techniques d'utilisation des sols fondamentalement différentes, et surtout un impact humain infiniment plus discret, n'ont sans doute pas permis de réaliser cet équilibre. II ne semble cependant pas que ces différences justifient à elles seules des points de vue aussi radicalement divergents à propos de la valeur écologique des fruticées sclérophylles sur le pourtour méditerranéen et en Californie.

En fait, les différences de structures dans l'architecture de la végétation signalées plus haut, nous paraissent, au moins en partie, justifier ces positions. En effet, du point de vue de l'érosion tout d'abord, la présence de nombreux chaméphytes prostrés et rampants ainsi que celle d'hémicryptophytes, assure indiscutablement une bien meilleure protection des horizons superficiels du sol en région méditerranéenne qu'en 
Californie méridionale où des rigoles d'érosion apparaissent partout dans les chaparrals entre les buissons érigés qui constituent la quasi totalité de leur végétation, et ce phénomène se manifeste même si l'inclinaison du sol est modeste et inférieure à 10 p. 100.

Ce facteur est sans doute très important puisque pour des régimes de pluies comparables et des précipitations horaires possibles aussi élevées dans les deux régions, il assure indiscutablement en région méditerranéenne, sur des matorrals même âgés, une meilleure protection à l'impact mécanique des gouttes, mais aussi une meilleure pénétration de l'eau dans le sol.

Il rend sans doute compte au moins en partie, du faible développement des horizons d'accumulation au niveau des sols sous les chaparrals et en particulier sous les chaparrals de faible altitude (Krammes, 1965).

Ce caractère de l'édaphisme paraît lié surtout à la faible importance d'apports végétaux rapidement humifiables qui sont par ailleurs emportés par l'érosion, et en particulier après les incendies (Debano et Conrad, 1976). Le développement fréquent en surface d'une couche non mouillable (Debano, 1969) accentue encore ces phénomènes. Remarquons qu'à notre connaissance jamais un phénomène de ce type n'a été signalé en région méditerranéenne de l'ancien monde.

\section{Les problèmes humains}

Il paraît bien difficile de ne pas considérer en région méditerranéenne, les maquis et garrigues, comme des formations essentiellement conditionnées par l'action de l'homme et surtout d'un pâturage quasi permanent, mais dont la valeur dynamique n'est pas nulle puisque presque partout, elles sont théoriquement susceptibles d'être transformées naturellement en forêts de conifères ou de chênes le plus souvent sclérophylles. Ce ne sont que sur les marges bioclimatiques extrêmes de la région méditerranéenne, en ambiance aride, que certains matorrals peuvent être considérés comme climax, encore que l'installation de Juniperus phœnicea en particulier y soit possible. En zone humide ou subhumide nous l'avons dit, l'existence de maquis denses climaciques peut aussi être envisagée, mais ils ne paraissent constituer que des cas très spéciaux. Partout ailleurs, sauf si le sol et la végétation sont définiłivement ruinés par des incendies répétés ou un pâturage excessif, ce qui est malheureusement le cas sur de vastes surfaces notamment en Espagne, au Maghreb, ou au Proche-Orient, la forêt méditerranéenne peut recoloniser les matorrals.

Il est très probable que cette particularité soit liée au dynamisme des essences locales mais on peut aussi se demander si elle ne découle pas également d'une adaptation plurimillénaire de la végétation à un type d'exploitation par l'homme et par les troupeaux, et en particulier à l'existence d'incendies périodiques et à un pâturage quasi permanent. Ceux-ci ont certainement facilité l'expansion des chaméphytes et des hémicryptophytes, diversifié la flore des fruticées en raison de l'ouverture permanente de sentes et de micro-clairières, mais aussi du piétinement important des sols, qui facilite lorsqu'il n'est pas exagéré, l'humidification des horizons supérieurs. La persistance générale de porte-graines épars est elle aussi importante.

C'est bien entendu ce mode d'exploitation, qui a maintenu de façon quasi géné- 
rale, l'extension des matorrals depuis au moins le Moyen-Age, et qui peut-être en a, en partie au moins, conditionné la composition floristique. De toute évidence ce type de végétation s'est révélé, dans des conditions raisonnables d'utilisation, constituer une réserve appréciable de nourriture pour les animaux, mais aussi une garantie très sûre contre l'érosion, sa conversion en forêt ou en pelouse étant toujours possible en fonction des modalités d'utilisation.

En Californie méridionale par contre, les choses sont bien différentes et il semble que les chaparrals ou du moins leurs constituants floristiques existent depuis la fin du Tertiaire au moins (Axelrod, 1973). La végétation n'a pas subi par ailleurs un impact important de la part de l'homme, la population indienne étant constituée essentiellement de chasseurs et de pêcheurs récoltant les fruits et les graines (Aschmann, 1959), pour lesquels l'incendie ne paraît pas avoir constitué une technique d'utilisation des sols. La colonisation massive aux siècles derniers a déterminé la mise en culture de nombreuses terres ef l'installation de troupeaux un peu partout, et il est probable, à notre avis, que le paysage actuel reflète encore cette intense déforestation des zones inférieures pour des raisons utilitaires, mais aussi la généralisation des techniques d'incendie pour régénérer les pâturages, comme cela se pratiquait couramment en Espagne.

Mais ici, la végétation s'est révélée extrêmement vulnérable à ces facteurs, notamment en raison du dynamisme des essences thermophiles bien plus restreint qu'en Europe, d'une dégradation des sols plus rapide en raison de l'inclinaison élevée des montagnes, de la prédominance des terrains granitiques, mais aussi des qualités intrinsèques de la flore locale.

C'est certainement alors que se sont mises en places les structures de végétation encore visibles actuel!ement, en particulicr cux altitudes inférieures: forêts localisées Jans les thalwegs, envahissemert sénéral des pentes par le chaparral.

Reste encore à discuter de la valeur dynamique actuelle de ce chaparral qui représente un écosystème où l'influence de l'homme n'apparaît guère que par le développement d'incendies périodiques, le parcours et le pâturage y étant actuellement quasi nuls, du moins en Californie méridionale. II est bien évident que cette question irritante n'a pas échappé aux botanistes et aux écologistes nord-américains. Cléments (1916) le considérait comme un sub-climnx artificiel lié à l'action du feu, et cette idée a longtemps prévalu. Toutefois, plus près de nous Horston (1951), puis Patric et Hanes (1964) sont arrivés à envisager la possibilité que ce chaparral, dans certaines conditions, puisse évolver vers un écosystème de type forestier. Il semble bien que cela soit en effet envisageable en dehors de l'incidence de feux, au niveau des chaparrals de moyenne altitude et localisés en exposition nord où dominent Quercus dumosa, Q. wislizenii, var. frutescens, Rhamnus crocea, Prunus ilicifolia, Lonicera subspicata, Rhus diversiloba, etc. Là en effet, les chênes en particulier peuvent dans les meilleurs cas atteindre 6 à $8 \mathrm{~m}$ (Patric et Hanes, 1964) et constituer de la sorte une véritable forêt. Au contraire en versant sud et sur les fortes pentes, la formation végétale dominée par Adenostoma fasciculatum et Salvia mellifera ne paraît en aucune façon, dans des conditions actuelles, susceptible d'évoluer vers un groupement forestier. Elle s'inclut effectivement dans une série dynamique particulière où le feu représente le facteur quasi unique d'évolution. 


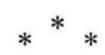

Que conclure de ces particularités?

Tout d'abord que les mêmes types de recherches n'ont pas été poursuivis sur le pourtour méditerranéen et en Californie. Les expérimentations, malheureusement fort onéreuses, réalisées aux U. S. A. sur l'écosystème chaparral se sont en effet révélées fort instructives, mais à quelques rares exceptions près n'ont pu être réalisées dans le bassin méditerranéen.

Inversement les analyses de structures effectuées de ce côté de l'Atlantique, grâce à des méthodes éprouvées, mais nécessitant une excellente connaissance de la flore ont été généralement négligées en Californie, si bien qu'il est souvent difficile de pousser très loin les comparaisons et à plus forte raison les analogies.

Quoi qu’il en soit, il apparaît à peu près certain que la flore et la végétation circum-méditerranéenne fortement influencée par l'homme et ses diverses activités depuis des millénaires, a particulièrement bien réagi à cette agression et s'y est adaptée. L'homme a gardé naturellement la possibilité d'orienter en définitive l'évolution des matorrals, évolution qui sauf dans les cas de surexploitation peut toujours s'effectuer vers la reconstitution de la forêt, en dehors de rares localités où les maquis paraissent paradoxalement réagir comme des chaparrals de Californie. Le rôle que jouent ces mêmes matorrals pour assurer la conservation des sols, de lutte contre l'érosion et l'équilibration du bilan hydrique du sol ne peut être mis en doute.

En Californie, par contre une végétation longtemps restée en situation marginale par rapport à l'action humaine, a été profondément modifiée, du moins dans la mise en place au sol des unités de végétation, au cours des deux derniers siècles. La situation est bien vite devenue dramatique aux altitudes inférieures puisque les forêts se sont vu confinées dans les thalwegs, et que partout ailleurs s'est installé un chaparral qui dans les conditions actuelles représente un écosystème à peu près totalement lié à l'apparition cyclique d'incendies et dont la valeur économique voire écologique est pratiquement nulle pour l'homme. II n'est en effet utilisé par lui, ni directement, ni indirectement et en particulier par l'intermédiaire des troupeaux, et de plus il ne joue qu'un rôle discret contre l'érosion servant tout au plus à assurer la stabilité des fortes pentes.

Il paraît actuellement difficile, en dehors d'une valeur esthétique indiscutable et qui ne doit pas être oubliée, d'envisager pour les chaparrals de Californie méridionale, une mise en valeur ou une utilisation, en dehors de coûteuses interventions humaines. Sa conversion en pâturages en particulier est possible et écologiquement justifiée, mais elle nécessite un entretien régulier par incendies contrôlés, et l'on manque encore de renseignements sur le rôle que pourrait y jouer un pâturage contrôlé notamment par des ovins. La reforestation des chaparrals de Californie du Sud ne paraît pas non plus avoir été sérieusement envisagée, sans doute parce qu'elle paraît vaine dans un pays aux ressources forestières considérables. II n'en reste pas moins que de nombreux problèmes d'utilisation et surtout de conservation du sol, des eaux et de la végétation se posent en Californie méridionale sans doute avec plus d'acuité encore que sur le pourtour de la Méditerranée. On peut toutefois se demander si des solutions seront proposées avant que l'expansion monstrueuse des villes, 
l'impact du smog, ou la mise en culture en terrasse sous irrigation à faible débit, ne détermine la ruine de ce type de végétation si remarquable et si singulier.

Reçu pour publication en juillet 1978.

\section{Summary}

Mediterranean «Matorrals» and Californian « Chaparrals». Some comparative aspects of their dynamics, structure and ecological significance

Matorrals and chaparrals show unquestionable physiognomic and even floristic homologies. However, noticeable differences appear when attempting to interpret the dynamic and structural values of these two vegetation types. This may account, at least partly, for the clear variance of opinion between Californian ecologists and those of the Old World as regards the analysis and methods of exploitation of these two vegetation types.

* P. QUEZEL is professor at the Faculty of Sciences and Techniques, Marseille-St Jérôme University.

\section{Références bibliographiques}

ASCHMANN H., 1973. Distribution and pecularity of Mediterranean Ecosystems, in Mediterranean Type Ecosystem, Ecological Studies 7, Springer Verlag Berlin Heidelberg, New York.

AXELROD D. I., 1973. History of the mediterranean ecosystem in California, in Mediterranean Type Ecosystem, Ecological Studies 7, Springer Verlag Berlin, 224-227.

BAGNOULS F. et GAUSSEN H., 1953. Saison sèche et indice xérothermique. Bull. Soc. Hist. Nat. Toulouse, 88, 193-239.

BARBOUR M. G. et Major J., 1977 . Terrestrial vegetation of California. A. Wiley Inter Sc. Publi. J. Wiley and Soon, New York.

BROWN T. C. ef BOSTER R. S., 1974. Effects of chaparral to grass conversion on wildfire suppression Costs. USDA Forest Service Research Paper, RM 119, 11 p.

CABLE D. R., 1975. Range management in the Chaparral Type and Ecological Basis. USDA Forest Service Research Paper, RM 155, 30 p.

CAMPBELL DH. et IRA L. W., 1947. Origins of the flora of California. Stanford Univ. Biol. SC, Stanford University Press, 20 p.

CLEMENTS F. E., 1916. Plant succession. Carnegie Inst. Publ. Washington, 242, 8 p.

CONRAD C. E. et DEBANO L. F., 1974. Recovery of southern California chaparral, ASCE National Meeting on water resources Engineering 21-24, Los Angeles.

COOPER W. S., 1922. The broad-sclerophyll vegetation of California. Carnegie Inst. of Washington Publ., 319, 124 p.

CORBETT E. S. et RICE R. M., 1966. Soil Slippage Increased by Brush Conversion, Glendora Pacific southwest Forest and Range experiment-Station, 8 p.

DAGET Ph., 1977. Le bioclimat méditerranéen, Caractères généraux modes de caractérisation. Vegetatio, 34, 1, 1-20.

DEBANO L. F., 1969. Water repellent soils : a worldwide concern in menagement of soil and vegetation. Agricultural Sciences Review, 7, 2, pp. 11-18.

DEBANO L. F. et CONRAD C. E., 1976. Nutrients lost in debris and runoff water from a burned chaparral watershed. Proceedings inter-agency sedimentation Conference, Denver, pp. 3-13 à 3-27.

DEBANO L. F. et RICE R. M., 1971. Fire in vegetation managements : its effects on soil. Amer. Soc. Civil Engineers, Symp. of Bozeman, 327-346.

DI CASTRI F. et MOONEY H. A., 1973. Mediterraneans Type Ecosystems. Ecological Studies 7, Springer Verlag, Berlin Heidelberg, New Yord, 405 p.

DUVIGNEAUD P., 1946. La variabilité des associations végétales. Bull. Soc. Bot. Belgique, 78, 107-134.

EHRIG F., 1973. Zum Problem der Macchien am Beispiel Korsikas. Mitt. Geograph. Gesell. München 58, 97-108.

EMBERGER L., 1952. Sur le quotient pluviothermique. C. R. Acad. Sc., 23, 2308-2310.

EMBERGER L., 1955. Une classification biogéographique des climats. Rec. Trav. Lab. Bot. Geol., Montpellier, 7, 3-43. 
GAUSSEN H., 1954. Théorie et classification des climats et microclimats. VIIe Cong. Int. Bot., Paris, 125-130.

GOLDBLATT P., 1977. An Analysis of Flora of Southern Africa, St-Louis. 34e Symposium floristique, African Systematic Studies.

GOUNOT M., 1969. Méthodes d'étude quantitative de la végétation, Masson Edit. 314 p.

HANES T. L., 1971. Succession after fire in the chaparral of Southern California. Ecol. Monogr., 41, pp. $27-52$.

HANES T. L., 1977. California chaparral, in Barbour et Major. Terrestrial vegetation of California, pp. 417-469.

HORSTON J. S., 1951. Some aspects of watershed management in southern California, U. S. Forest Service, California forests and range expt. Stat. Misc. Paper I, 10-17.

HORTON J. S. et KRAEBEL C. J., 1955. Development of vegetation after fire in the chamise chaparral in southern California. Ecology, 36, pp. 244-262.

JOHNSON A. W., 1973. Historical view of the concept of Ecosystem convergence, in Mediterranean type ecosystems, Springer Verlag.

KRAMMES J. S., 1965. Seasonnal debris movement from steep mountainside slopes in southern California. Proc. Federal Interagency sedimentation Conference, U. S. Dept. Agr. Misc. Publ. 970, 806-810.

KUNHOLTZ-LORDAT G., 1938. La terre incendiée, essai d'agronomie comparée, La Maison Carrée, ed. Nimes.

MC PHERSON J. K. et MULLER C. H., 1969. Allelopatics effects of Adenostema fasciculatum in the California chaparral. Ecol. Monog., 39, pp. 177-198.

MOONEY H. A. et PARSONS D. J., 1973. Structure and function of California chaparral, an exemple from San Dimas. Mediterranean type Ecosystems edited by F. di Castri et H. Mooney, Springer Verlag, Berlin Heidelberg, New York, pp. 83-113.

NAHAL I., 1976. La variabilité des caractéristiques biologiques des climats méditerranéens arides. Acta Ecologica Iranica 1, 35-48.

PATRIC J. H. et HANES T. L., 1964. Chaparral succession in a San Gabriel Mountain area of California. Ecology, 45, 2, 253-360.

QUEZEL P., 1976. Les forêts du pourtour méditerranéen, in Forets et Maquis méditerranéens. Notes Techniques, MAB 2, U.N.E.S.C.O., 9-34.

QUEZEL P., 1977. Floristic Analysis of Mediterranean and Saharian Africa : Historical interpretation of flora. St-Louis. 34e Symposium floristique, African Systematic Studies.

RAVEN P. H., 1971. The relationships between mediterranean Flores, in Plant Life of S-W Asia, Edinburg, Bot. Soc.

RICE R. M., 1973. The hydrology of chaparral watersheds, Proceedings Symp. on living with the chaparral, Riverside.

ROWE P. B., 1963. Streamflow increases after removing woodland-riparian vegetation from a southern california watershed. Journ. Forestry, 61, pp. 365-370.

SAMPSON A. W., 1944. Plant succession on burned chaparral lands in northern California, Univ. of California Bull., 685 Berkeley, 144 p.

SAUVAGE Ch., 1961. Recherches botaniques sur les suberaies marocaines. Trav. Bot. Inst. Sc. Chérifien, 21, 1-462.

SAUVAGE Ch., 1963. Le coefficient pluviométrique d'Emberger son utilisation ef la représentation graphique de ses utilisations au Maroc, Ann. Ser. Phys. Inst. Sc. Chérifien, 20, 11-23.

TOMASELLI R., 1976. La dégradation du maquis méditerranéen, in Forêts et maquis méditerranéens. Notes Techn. MAB 2, U.N.E.S.C.O., 35-76.

ZINKE P. J., 1965. Mediterranean analogs of California soil vegetation types. Berkeley Univ. Wildland Research Center.

ZINKE P. J., 1973. Analogies between soil and vegetation types of Italy, Greece and California, in Mediterraneans types Ecosystems, Springer Verlag Berlin Heidelberg, New York, 61-82. 